\title{
An Enhanced Fault Tolerant Control Against Current Sensor Failures in Induction Motor Drive by Applying Space Vector
}

\author{
Cuong Dinh TRAN ${ }^{1,3}$, Pavel BRANDSTETTER ${ }^{3}$, Minh Chau Huu NGUYEN ${ }^{3}$, \\ Sang Dang $H^{1,3}$, Phuong Nhat PHAM ${ }^{1,3}$, Bach Hoang DINH ${ }^{2, *}$ \\ ${ }^{1}$ Faculty of Electrical and Electronics Engineering, Ton Duc Thang University, \\ Ho Chi Minh City, Vietnam \\ ${ }^{2}$ Power System Optimization Research Group, Faculty of Electrical and Electronics Engineering, \\ Ton Duc Thang University, Ho Chi Minh City, Vietnam \\ ${ }^{3}$ Faculty of Electrical Engineering and Computer Science, VSB-Technical University of Ostrava,
} Czech Republic

*Corresponding Author: Bach Hoang DINH (Email: dinhhoangbach@tdtu.edu.vn)

(Received: 16-Nov-2019; accepted: 3-Feb-2020; published: 31-March-2020)

DOI: http://dx.doi.org/10.25073/jaec.202041.269

\begin{abstract}
In this paper, an enhanced active fault-tolerant control (FTC) is proposed to solve a current sensor failure in the induction motor drive (IMD) using two current sensors. The proposed FTC method applies only one observer to diagnose the faults and reconfigure the control signals by the space stator current. The diagnosis function is made up of a comparison algorithm between the measured current space vector and the estimated space vector. Then, incorrect feedback stator currents are replaced by the estimated values in the reconfiguration function. The amplitude of a healthy measured current is applied to adjusted the accuracy of estimated current signals. The IMD uses the field-oriented control (FOC) technique to control the speed and torque. The effectiveness in stabilizing the IMD system when a current sensor error occurs is verified by various simulations in the Matlab-Simulink environment.
\end{abstract}

\section{Keywords}

Diagnosis, estimated currents, faulttolerant control, FOC, IMD.

\section{Nomenclature}
$\Psi_{S}^{S} \quad$ Stator flux vector in $[\alpha, \beta]$ coordinate system
$\Psi_{R}^{S} \quad$ Rotor flux vector in $[\alpha, \beta]$ coordinate system
$i_{S} S \quad$ Stator current vector in $[\alpha, \beta]$ coordinate system
$i_{R}{ }^{S} \quad$ Rotor current vector in $[\alpha, \beta]$ coordinate system
$u_{S} S \quad$ Stator voltage vector in $[\alpha, \beta]$ coordinate system

$u_{S \alpha}, u_{S \beta} \quad$ Stator voltage component in $[\alpha, \beta]$ system

$u_{S x}, u_{S y} \quad$ Stator voltage component in $[x, y]$ system

$u_{a}, u_{b}, u_{c} \quad$ Stator voltage component in $[a, b, c]$ system 


$\begin{array}{ll}i_{S x} & \text { Flux current component } \\ i_{S y} & \text { Torque current component } \\ R_{S}, R_{R} & \text { Stator and rotor resistance } \\ L_{S}, L_{R} & \text { Stator and rotor inductance } \\ L_{m} & \text { Magnetizing induction } \\ T_{R} & \text { Rotor time constant } \\ \omega_{m} & \text { Mechanical angular speed } \\ p & \quad \text { Pole pair number } \\ \psi_{R}, \gamma & \quad \begin{array}{l}\text { Nominal rotor flux and rotor } \\ \text { flux angle }\end{array}\end{array}$

\section{Introduction}

Nowadays, with diverse applications in the industrial field, induction motors (IMs) are essential equipment in the manufacturing industry. Due to the development of power electronics devices combining modern control algorithms, the IMD is almost capable of replacing the DC motor drive in industrial applications requiring high stability and safety. The application of modern control models plays a vital role in ensuring IDMs operation stably with high accuracy. Among many control methods, the field-oriented control (FOC) algorithm is considered as a typical representation by its robustness and precision in controlling speed and torque of the IMD [1].

The feedback signals of sensors, such as the speed encoder, current sensors, voltage sensors, play a crucial role in the control-loop of IMDs. Unexpected sensor failures can weaken the performance of IMDs, and if not handled promptly, they can cause a collapse of the IMD system [2]. In modern models using VSI-fed, the signals of voltage sensors are often replaced by combination dc-link voltage and switching inverter, and IMD [3]. The classical diagnosis method based on the comparison of the measured speed signal and estimated value works well with the speed sensor fault [3]-[5]. Therefore, the failures of voltage sensors and a speed sensor have not been considered in this paper.

The three-phase stator current is a vital element to calculate the control signals in the closed-loop of the IMD system. The incorrectness of these signals can affect the stability and reliability of ID drive systems seriously. Many fault-tolerant-control (FTC) methods have been researched to provide solutions for this problem.

For the IMDs using three-sensors to generate feedback current signals, the common approach of the FTC method is based on Kirchhoff's law. The failure of a sensor can be determined by the non zero value of the total three measured phase current. In paper [6], the authors used three observers to detect current faults. Each of the observers receives the two currents from different two current sensors to transform the stator currents in $[a, b, c]$ become to space vector into the stationary coordinate system $[\alpha, \beta]$. The comparison algorithms between the estimated stator current and stator current space vector of each observer are implemented to determine the minimum error. As a result, a faulty current sensor is located by the observer corresponding to the minimum error. Then a healthy observer provides the stator current signals to the controller of IMD.

Due to the advantages of cost and size machine, in modern IMD systems, only two sensors are used to provide the feedback current signals to control-loop. Here, Kirchhoff's law is not applied to diagnose current failures. In paper [3], a current-factor for each phase is proposed, which is the difference between the peak value of the measured and estimated current in an electrical cycle. In a healthy condition, the current-factor equal to zero value for both phases. If the difference of a current factor is higher than a particular threshold, that means the failure of a sensor is detected. Then this sensor is isolated, and estimated signals are applied to replace the wrong signals.

In [7], the authors proposed the new idea is to apply observers corresponding to different coordinate systems $[\alpha, \beta]$, and each of them is offset 120 degrees from others. Each observer receives the voltages and one phase-current of the corresponding sensor. The voltage signals 
in a three-phase coordinate system $[a, b, c]$ were transformed into corresponding observers, and then the estimated stator currents were calculated from these voltages. The $\alpha$-component of the estimated-current in each observer is parallel, and have the same amplitude with corresponding measured stator current. Based on the comparison between estimated currents' $\alpha$ component and the measured-current, the failure of each current sensor can be diagnosed precisely. Finally, the wrong current signal of the sensor is replaced by estimated-currents of the suitable observer.

In [8], two diagnosis techniques are presented to generate the current-residuals dynamics in the coordinate systems $[\alpha, \beta]$ : the differentialalgebraic estimation of fault-dynamics and the other method based on a combination of the Robust Integral Sign of the Error observer. In the first method, the residual of Algebraic Fault Estimator is compared to two thresholds to diagnose the current sensor fault. The idea of the last method is to determine the differentialalgebraic-estimation of the faults-current residuals based on the estimated currents. The advantages of these methods are the capability to detect single and multiple sensor faults. However, the diagnosis-algorithms are complex, and testthresholds are severely affected by the change of machine parameters.

In this paper, we proposed an upgrading fault tolerance control against the total failure of current sensors in the IMD system, which applies two current sensors. In this method, the only current-observer is applied to detect the failure of current-sensors. Besides, the healthy-current also is used to adjust the correction of the estimated current. After identifying the currentfault, the estimated-current can be used to replace the wrong measured signal. Because the algorithm of the proposed method is simple, the FTC function works faster than the traditional methods. The signal of the faulty current sensor is isolated and replaced by the suitable estimated current quickly, and the IMD operates stably again. The simulation results have substantiated the effectiveness of the proposed method against the current-faults.

\section{Fault tolerant control for current sensor}

This section presents a dynamic model of the IM using the FOC technique. Then the proposed space vector method is compared with the transformation method to prove capability against a current sensor fault.

\subsection{The dynamic model of IM and FOC technique}

The primary function of an IM is to control speed and torque according to the requirements in specific applications. The dynamic model of an IM is expressed by the relationship between voltage, current, and magnetic flux in the stationary coordinate system $[\alpha, \beta]$ as below equations:

$$
\begin{gathered}
u_{S}^{S}=R_{S} i_{S}^{S}+\frac{d \Psi_{S}^{S}}{d t} \\
0=R_{R} i_{R}^{S}+\frac{d \Psi_{R}^{S}}{d t}-j p \omega_{m} \Psi_{R}^{S} \\
\Psi_{S}^{S}=L_{S} i_{S}^{S}+L_{m} i_{R}^{S} \\
\Psi_{R}^{S}=L_{m} i_{S}^{S}+L_{R} i_{R}^{S}
\end{gathered}
$$

Based on IM's model, the FOC method is applied to control the IMD. The primary principle in the FOC technique is a separate control between torque and rotor flux at the same time. In the rotating coordinate system $[x, y]$, the stator current space vector is separated into two perpendicular elements, as shown in Fig. 1. By the way, the rotor flux is controlled by the $i_{\mathrm{S}_{x}}$, and the electrical torque is controlled by the $i_{\mathrm{S}_{y}}$ $[9,10]$.

The stator current signals in a three-phase coordinate system $[a, b, c]$ can be transformed into a two-phase coordinate system $[\alpha, \beta]$ by Clarke's formulas as [10]:

$$
\left[\begin{array}{c}
i_{S \alpha} \\
i_{S \beta}
\end{array}\right]=\frac{2}{3}\left[\begin{array}{rrr}
1 & -\frac{1}{2} & -\frac{1}{2} \\
0 & \frac{\sqrt{3}}{2} & -\frac{\sqrt{3}}{2}
\end{array}\right]\left[\begin{array}{l}
i_{a} \\
i_{b} \\
i_{c}
\end{array}\right]
$$




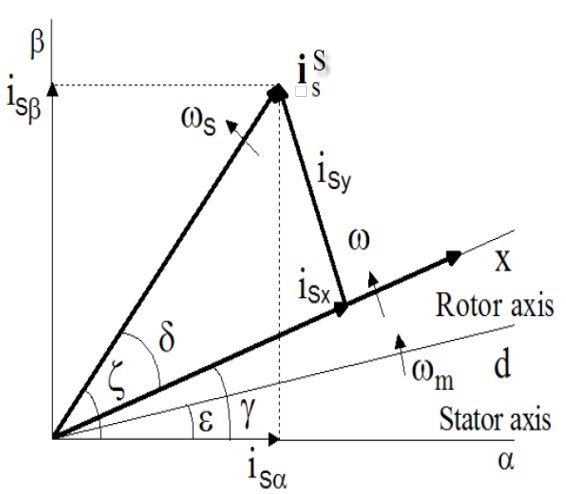

Fig. 1: Vector diagram of the FOC technique.

Here, $i_{c}=-i_{a}-i_{c}$.

By the above formula, the amplitude of the stator current space vector equals the peak of each stator current in the three-phase coordinate system $[a, b, c]$. Next step, the $[\alpha, \beta]$ stator current is transformed to coordinate system $[x, y]$ by Park's formulas as below [10]:

$$
\left[\begin{array}{c}
i_{S x} \\
i_{S y}
\end{array}\right]=\left[\begin{array}{cc}
\cos (\gamma) & \sin (\gamma) \\
-\sin (\gamma) & \cos (\gamma)
\end{array}\right]\left[\begin{array}{c}
i_{S \alpha} \\
i_{S \beta}
\end{array}\right]
$$

Here, $\gamma$ is the angle of rotor flux.

By applying the FOC technique, we can control the electrical torque and the rotor speed by the relationship with two $[x, y]$ current components, as the below formulas:

$$
\begin{aligned}
& i_{S y}=\frac{2}{3 p} \frac{L_{R}}{L_{m}} \frac{T_{e}}{\psi_{R}} \\
& \psi_{R}=\frac{L_{m}}{T_{R}+1} i_{S x}
\end{aligned}
$$

From the above dynamic model, the control structure of IMD based on the FOC technique is described, as in Fig. 2. Here: " $R_{x}$ " is the block of the integrator, "Ri $i_{x}$ " is the block of PI controller, "BVN" is the block of vector rotation, and "BZV" is the block of Decoupling. The feedback signals from two current sensors and the speed sensor are used to estimate the modulus of magnetizing current and the angle of rotor flux by the Current-model of IM [11, 12], as in (7)-(9).

$$
\psi_{R}^{S}=\int\left[\left(j \omega_{m}-\frac{1}{T_{R}}\right) \psi_{R}^{S}+\frac{1}{T_{R}} L_{m} i_{S}^{S}\right] d t
$$

$$
\begin{aligned}
& \psi_{R \alpha}=\int\left[-\frac{1}{T_{R}} \psi_{R \alpha}-\omega_{m} \psi_{R \beta}+\frac{1}{T_{R}} L_{m} i_{S \alpha}\right] d t \\
& \psi_{R \beta}=\int\left[-\frac{1}{T_{R}} \psi_{R \beta}+\omega_{m} \psi_{R \alpha}+\frac{1}{T_{R}} L_{m} i_{S \beta}\right] d t
\end{aligned}
$$

Then, the angle of rotor flux is used to transform $[\alpha, \beta]$-currents to $[x, y]$-currents by the equation (6). By the way, we can obtain two primary elements $" i_{\mathrm{S}_{x}}, i_{\mathrm{S}_{y}}$ " to control the torque and rotor speed of IM separately. By applying the decoupling circuits in rotating coordinate system $[x, y]$, two control current " $i_{\mathrm{S}_{x}}, i_{\mathrm{S}_{y}}$," combining with IM's parameters generate reference voltages for controlling the switching inverter by the sine PWM method.

As a result, the electrical torque and rotor speed are controlled independently by two " $i_{\mathrm{S}_{x}}$, $i_{\mathrm{S}_{y}}$ " elements in FOC-loop.

\subsection{Fault-tolerant control against current sensor faults by the axes transformation method}

This method is described in the paper [7]. The main idea of this FTC is to use control-scheme integrates different observers, taking one-phase measured current as inputs. Each observer receives the voltages and one phase-current of the corresponding sensor. The voltage signals in a three-phase coordinate system $[a, b, c]$ were transformed into a stationary coordinate system $[\alpha, \beta]$ corresponding observers, as in (12), (13)

$$
\begin{aligned}
& u_{S \alpha}^{a}=\frac{2 u_{a}-u_{b}-u_{c}}{3} ; u_{S \beta}^{a}=\frac{u_{b}-u_{c}}{\sqrt{3}} \\
& u_{S \alpha}^{b}=\frac{2 u_{b}-u_{c}-u_{a}}{3} ; u_{S \beta}^{b}=\frac{u_{c}-u_{a}}{\sqrt{3}}
\end{aligned}
$$

Then the estimated currents were estimated in the corresponding coordinate $[\alpha, \beta]$. The $\alpha$-current-component in each observer is the same amplitude with the corresponding measured current. Based on the comparison between estimated currents' $\alpha$-component and the 


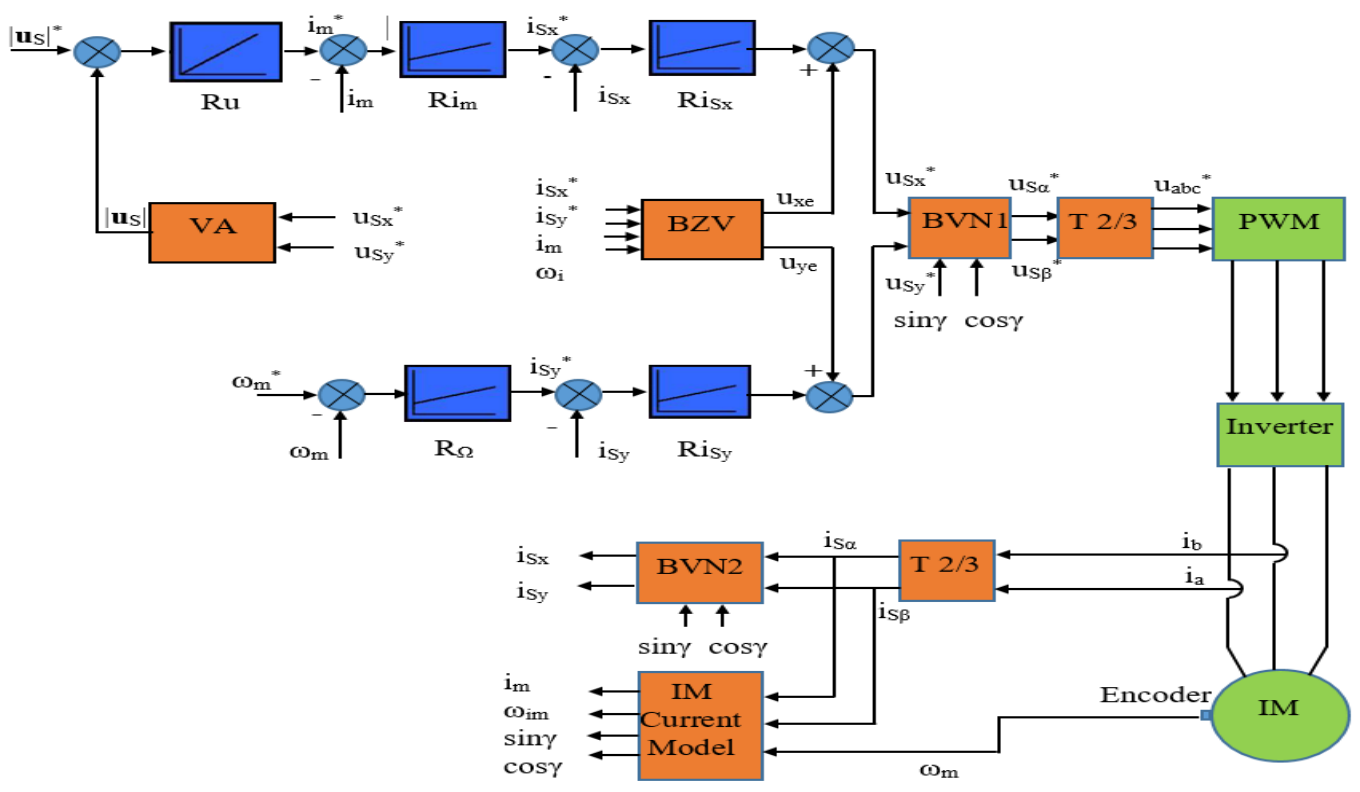

Fig. 2: Control block diagram applying the FOC technique.

measured-current, the failure of each current sensor can be located, as shown in (14).

$$
F_{\text {current_i }}{ }_{i}=\left|\hat{i}_{S \alpha}-i_{i}\right|>\text { Threshold }
$$

Finally, the wrong current signal of the sensor is replaced by estimated-currents of the suitable observer.

The current error of estimated signals is corrected by the deviation between $\alpha$ estimated current and corresponding measured current, illustrated as Fig. 3.

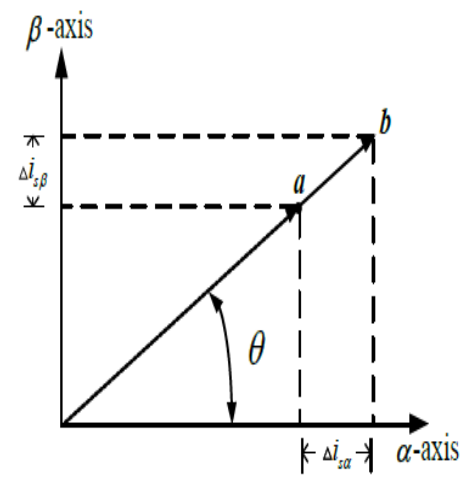

Fig. 3: Deviation between estimated and measured current.
The correction factor $\Delta i_{S \beta}=\Delta i_{S \alpha} \cdot \tan \theta$. Here $\theta$ is the angular of the estimated-current in the corresponding coordinate system $[\alpha, \beta]$, and $\tan \theta=i_{S \beta} / i_{S \alpha}$. To prevent undefined values of $\tan \theta$ when $\theta$ approaches to $90^{\circ}$ and $270^{\circ}$, the authors propose $\tan \theta\left(89^{\circ}<\theta<91^{0}, 269^{\circ}<\right.$ $\left.\theta<271^{0}\right)$ is limited to 57 or $-57\left(\tan 89^{\circ}=57\right.$, $\left.\tan 91^{\circ}=-57\right)$.

\subsection{Fault-tolerant control against current sensor faults by the proposed space vector method}

As mentioned above, the feedback current signals play a decisive role in controlling the IMD. In normal operation mode, the feedback signals are provided by two current sensors. When one failure of the sensor occurs, these signals are incorrect, which leads the FOC controller can work wrongly. Therefore, it is necessary to have an FTC system integrated into the control unit to ensure the system operates stably and reliably.

FOC method, including FTC function, is presented in the block diagram, as shown in Fig. 4. 


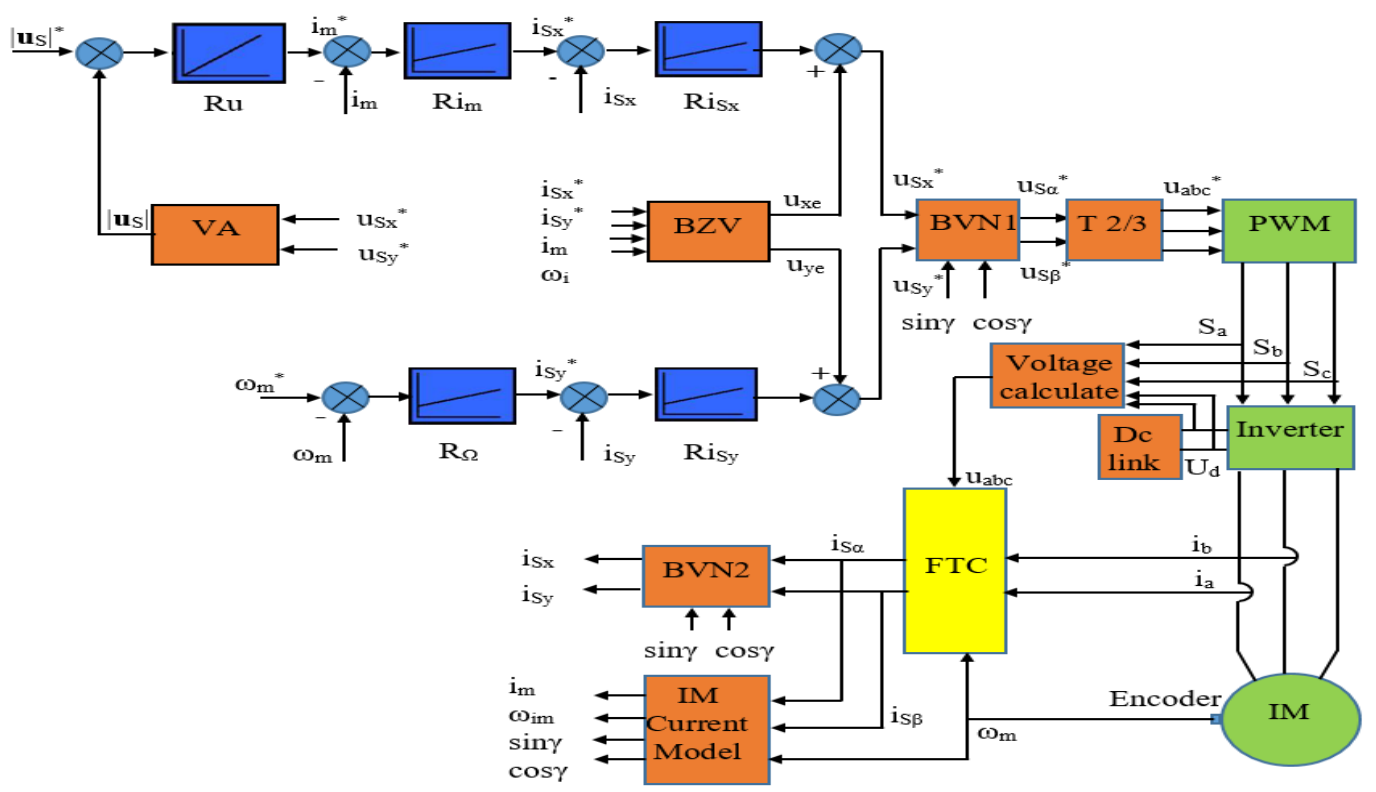

Fig. 4: Control block diagram applying FOC, including FTC function.

Here, the outputs of the FTC unit are alpha, beta components of measured stator current if the current sensors are in good condition, and of estimated current when a current sensor failure occurs.

The inputs of the FOC controller includes the stator voltages $\left[u_{S \alpha}, u_{S \beta}\right]$ calculated from switching inverter signals and DC links, the measured currents from sensors, the rotor speed. The FTC unit performs an analysis of the operating status and provides suitable control signals back to the FOC controller.

The proposed FTC process consists of two steps: the diagnosis step and reconfiguration step.

Fault diagnosis step: An error of the current sensor is diagnosed by comparing the modulus between estimated and measured stator current space vector.

The $\alpha, \beta$ components of measured current are determined by the formula (5), and then, the modulus of measured current space vector can be calculated as the below formula:

$$
\left|i_{S_{-} m}^{S}\right|=\sqrt{i_{S \alpha_{-} m}^{2}+i_{S \beta_{-} m}^{2}}
$$

The estimated current can be derived from the voltage signals, the rotors speed, and the IM's parameter [13, 14] as follows:

$$
\begin{aligned}
\frac{d \hat{i}_{S}^{S}}{d t} & =K\left[u_{S}^{S}-\left(R_{S}+j \frac{L_{m}^{2}}{L_{R}} \omega_{R}\right) \hat{i}_{S}^{S}\right. \\
& \left.+\left(\frac{L_{m} R_{R}}{L_{R}}-j L_{m} \omega_{R}\right) \hat{i}_{R}^{S}\right]
\end{aligned}
$$

$$
\begin{aligned}
\frac{d \hat{i}_{R}^{S}}{d t} & =K\left[\left(\frac{1}{L_{S}} u_{S}^{S}-\left(\frac{R_{S}}{L_{S}}+j \omega_{R}\right) \hat{i}_{S}^{S}\right.\right. \\
& \left.+\left(\frac{R_{R}}{L_{m}}-j \frac{L_{R}}{L_{m}} \omega_{R}\right) \hat{i}_{R}^{S}\right]
\end{aligned}
$$

Here: $K=\frac{L_{R}}{L_{S} L_{R}-L_{m}^{2}} ; \omega_{R}=p \omega_{m}$

The estimated algorithms are improved the accuracy by addition of two correction-factors, as follows:

$$
\frac{d \hat{i}_{S \alpha}}{d t}=K\left[\begin{array}{c}
u_{\alpha}-R_{S} \hat{i}_{S \alpha} \\
+\frac{L_{m}^{2}}{L_{R}} \omega_{R} \hat{i}_{S \beta} \\
+\frac{L_{m} R_{R}}{L_{R}} \hat{i}_{R \alpha} \\
+L_{m} \omega_{R} \hat{i}_{R \beta}
\end{array}\right]+f(c o)
$$




$$
\begin{gathered}
\frac{d \hat{i}_{S \beta}}{d t}=K\left[\begin{array}{l}
u_{\beta}-R_{S} \hat{i}_{S \beta} \\
-\frac{L_{m}^{2}}{L_{R}} \omega_{R} \hat{i}_{S \alpha} \\
-L_{m} \omega_{R} \hat{i}_{R \alpha} \\
+\frac{L_{m} R_{R}}{L_{R}} \hat{i}_{R \beta}
\end{array}\right]+f(s i) \\
\frac{d \hat{i}_{R \alpha}}{d t}=\left[\begin{array}{l}
\frac{1}{L_{S}} u_{\alpha}-\frac{R_{S}}{L_{S}} \hat{i}_{S \alpha} \\
+\omega_{R} \hat{i}_{S \beta}+\frac{R_{R}}{L_{m}} \hat{i}_{R \alpha} \\
+\frac{L_{R}}{L_{m}} \omega_{R} \hat{i}_{R \beta}
\end{array}\right] \\
\frac{d \hat{i}_{R \beta}}{d t}=K\left[\begin{array}{l}
\frac{1}{L_{S}} u_{\beta}-\omega_{R} \hat{i}_{S \alpha} \\
-\frac{R_{S}}{L_{S}} \hat{i}_{S \beta} \\
-\frac{L_{R}}{L_{m}} \omega_{R} \hat{i}_{R \alpha} \\
+\frac{R_{R}}{L_{m}} \hat{i}_{R \beta}
\end{array}\right]
\end{gathered}
$$

The deviation between measured and estimated current is calculated, as below:

$$
f=\left.|| \hat{i}_{S}^{S}|-| i_{c}\right|_{\text {peak }} \mid
$$

Here:

$+\left|i_{c}\right|_{\text {peak }}$ : the peak value of phase C- sinusoidal current;

$+\hat{i}_{S}^{S}$ : the modulus of estimated current, corresponding Eqs. (18), (19);

The correction-factors " $\mathrm{f}(\mathrm{co}), \mathrm{f}(\mathrm{si})$ " are determined as (23)

$$
\left\{\begin{array}{l}
f(c o)=\left\{f \cdot \cos \theta \& F_{\text {current }}\right\} \\
f(s i)=\left\{f \cdot \sin \theta \& F_{\text {current }}\right\}
\end{array}\right.
$$

where: $F_{\text {current }}$ : indication-flag of current sensor fault

Diagnosis function can be determined by the comparison algorithm between the modulus of measured and the estimated current space vector, as follows:

$$
\begin{aligned}
& I F:|| i_{S_{-}}^{S} m|-| \hat{i}_{S}^{S}|| \geqslant T_{\text {threshold }} \\
& \text { THEN }: F_{\text {current }}=1 ; \text { ELSE }: F_{\text {current }}=0 .
\end{aligned}
$$

$\mathrm{T}_{\text {threshold }}$ : is a maximum difference between the amplitude of measured current space vector and magnitude of the estimated current space vector. In this paper, $\mathrm{T}_{\text {threshold }}$ is proposed as a $10(\%)$ value of rated current.

Reconfiguration step: after the total-currentfailure is detected, the FTC controller isolates the wrong feedback signals of current sensors, and replace them by the estimated current signs. As a result, the IMD operates stably with the estimated current signals, which are corrected by the remain healthy current sensor.

Finally, the FTC unit determines the fault state and provides the feedback current to the FOC controller as the flowchart in Fig. 5:

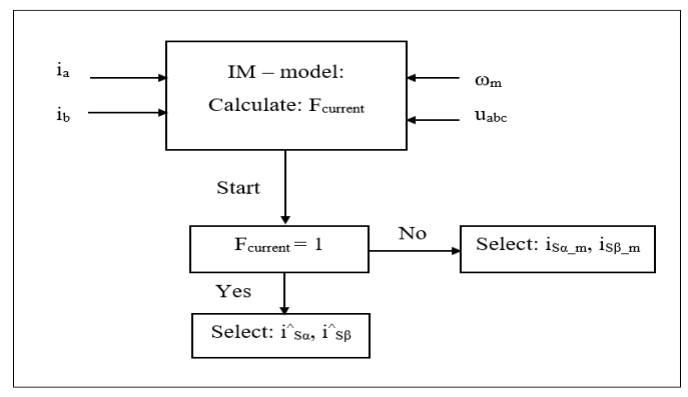

Fig. 5: Flowchart of FTC unit's function.

\section{Simulation results}

In this part, simulations in Matlab/Simulink have been implemented and compared between the axes transformation method and space vector method. Here, the model induction motor with machine parameters listed in Tab. 1.

The simulations have been implemented at the normal reference speed zone. The IMD is controlled by the FOC technique in both healthy sensor conditions and false conditions. The con- 
Tab. 1: The main parameters of IM

\begin{tabular}{|c|c|c|}
\hline $\begin{array}{c}\text { Description: } \\
\text { Symbol }\end{array}$ & Value & Unit \\
\hline Rated Power: $\mathrm{P}_{n}$ & 2.2 & $\mathrm{~kW}$ \\
\hline Rated Voltage: $\mathrm{U}_{n}$ & 400 & $\mathrm{~V}$ \\
\hline Rated Torque: Tn & 14.8 & $\mathrm{~N} . \mathrm{m}$ \\
\hline Rated speed: $n_{n}$ & 1420 & $\mathrm{rpm}$ \\
\hline Rated stator current: $\mathrm{I}_{n}$ & 4.85 & $\mathrm{~A}$ \\
\hline Stator resistance: $\mathrm{R}_{S}$ & 3.179 & $\Omega$ \\
\hline Rotor resistance: $\mathrm{R}_{R}$ & 2.118 & $\Omega$ \\
\hline Mutual inductance: $\mathrm{L}_{m}$ & 0.192 & $\mathrm{H}$ \\
\hline Pole pair number: $\mathrm{p}$ & 2 & - \\
\hline Stator inductance: $\mathrm{L}_{S}$ & 0.209 & $\mathrm{H}$ \\
\hline Rotor inductance: $\mathrm{L}_{R}$ & 0.209 & $\mathrm{H}$ \\
\hline Rated Rotor flux: $\Psi_{\mathrm{Sn}}$ & 0.757 & $\mathrm{~Wb}$ \\
\hline
\end{tabular}

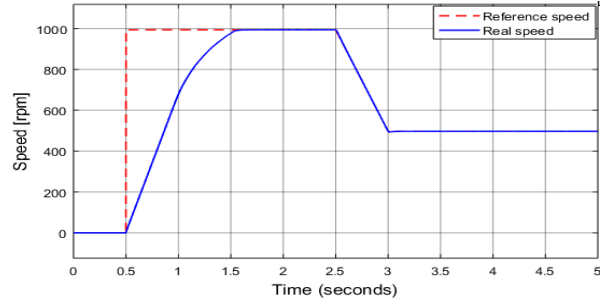

(a)

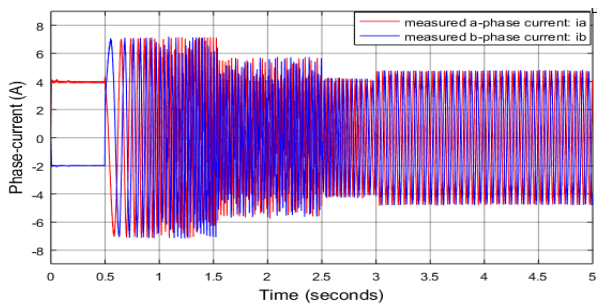

(b)

Fig. 6: Nominal speed range: (a) reference speed and measured speed, (b) two-phase measured current signals in healthy current sensors condition.

trol structure of the IMD is the same in both two FTC methods.

The IMD has been operated according to the reference rotor speed in two ranges: the nominal speed and low-speed, as shown in Fig. 6(a) and Fig. 7(a). A constant load torque equal to $5 \mathrm{~N} . \mathrm{m}$ was applied at $0.5 \mathrm{~s}$ time during the operation process. Reference speed set up to a value $70(\%)$ of rated speed $t=0.5 \mathrm{sec}$, and then, it

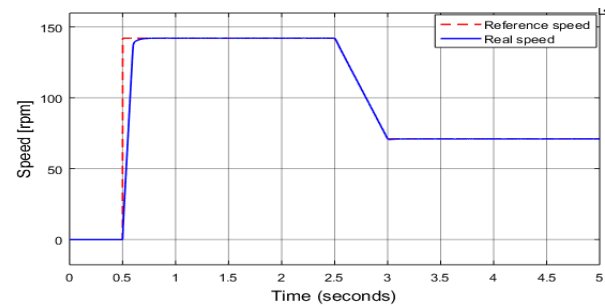

(a)

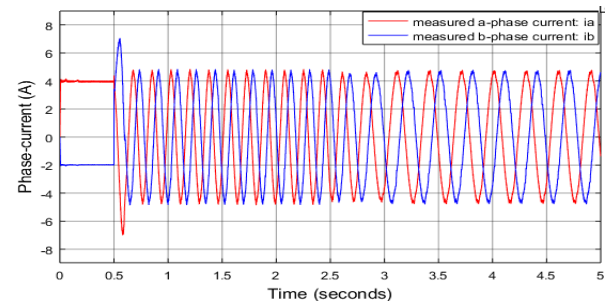

(b)

Fig. 7: Low-speed range: (a) reference speed and measured speed, (b) two-phase measured current signals in healthy current sensors condition.

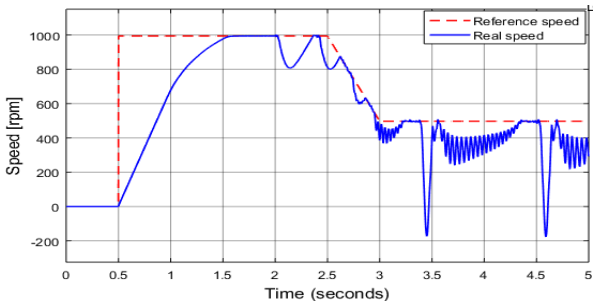

(a)

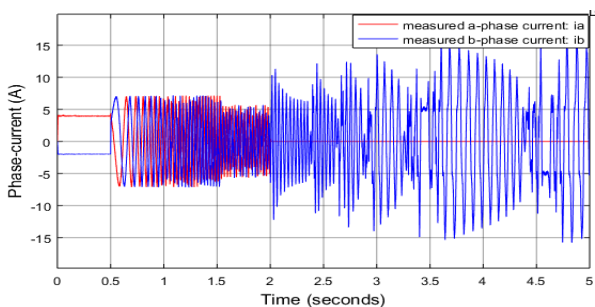

(b)

Fig. 8: Nominal speed range: (a) reference speed and measured speed, (b) two-phase measured current signals with "A phase" current sensor fault.

set down to $35 \%$ of the rated value following the slant line $(2.5 \mathrm{sec}-3.0 \mathrm{sec})$. The two measured stator currents in a healthy condition are 


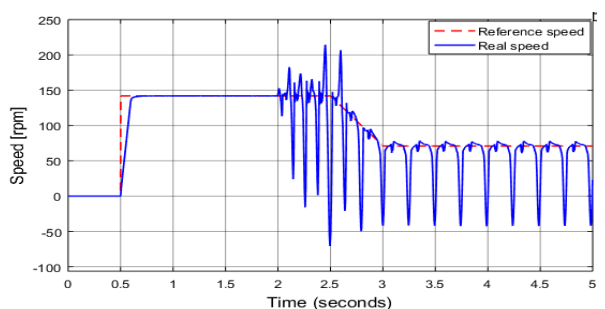

(a)

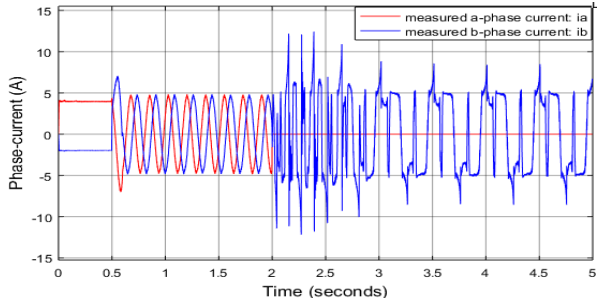

(b)

Fig. 9: Low-speed range: (a) reference speed and measured speed, (b) two-phase measured current signals with "A phase" current sensor fault.

also shown in Fig. 6(b). Similarly, the reference speed is set at $10 \%$ and $5 \%$ of the rated speed in the low-speed area as Fig. 7(b). The IMD applying the FOC controller operates stably during the steady-state and transient state in both cases above.

When a total failure of the current sensor occurs at $t=2.0 \mathrm{sec}$, the value of feedback "A phase" current back to zero that leads the FOC controller operates wrong. As a result, the IMD becomes unstable and collapses, as shown in Fig. 8 and Fig. 9. Therefore, the purpose of FTC methods is to maintain the stability of the drive system, even under fault operating conditions.

In the first case, the FTC function of the axes transformation method against a current sensor fault is simulated by Matlab/Simulink. Fig. 10(a) and Fig. 11(b) depicted a failure of Aphase current occurrence, and feedback current signal equals to zero. The fault-location function set to a high level to detect the false as in Fig. 10(b) and Fig. 11(b). The rotor speed slightly fluctuates in a short time, and after the incorrect current signals are replaced by the es-

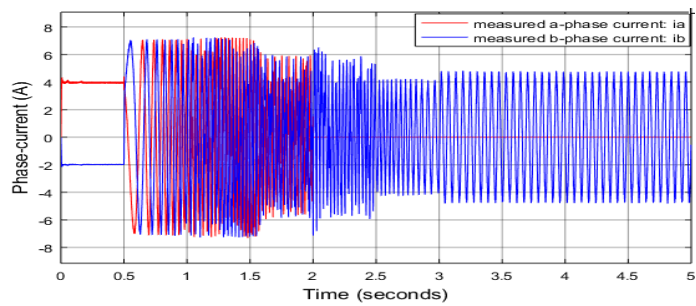

(a)

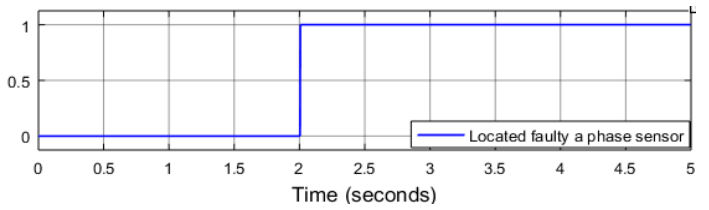

(b)

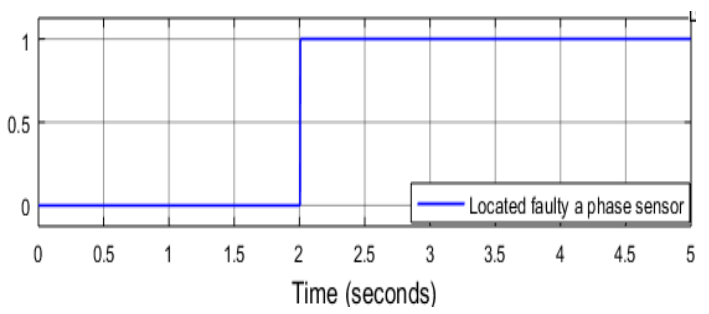

(c)

Fig. 10: Nominal speed range: IMD applying axes transformation-FTC method against a current sensor fault: (a) two-phase measured current, (b) fault indication function, (c) rotor speed.

timated value, the IMD works stably again, as in Fig. 10(c) and Fig. 11(c).

Next step, the simulations have been implemented to demonstrate the effectiveness of the proposed FTC method. Similar to the above case, Fig. 12(a) and Fig. 13(a) shows a current sensor fault with A-phase. After the current fault occurrence, the fault diagnosis of the proposed method works immediately, as in Fig. 12(b) and Fig. 13(b). The wrong signals are isolated and replaced by the estimated current, which is corrected by the healthy current sensor. Fig. 12(c) and Fig. 13(c) demonstrated that the IMD still operates reliably, even under the sensor fault condition.

By comparison between two methods, we can recognize that the detection time of the proposed 


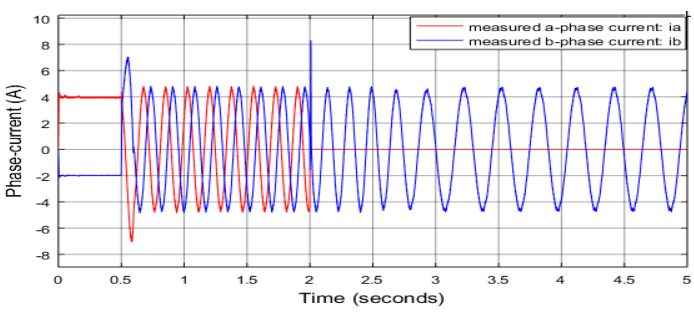

(a)

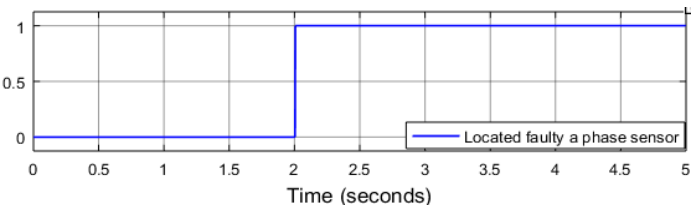

(b)

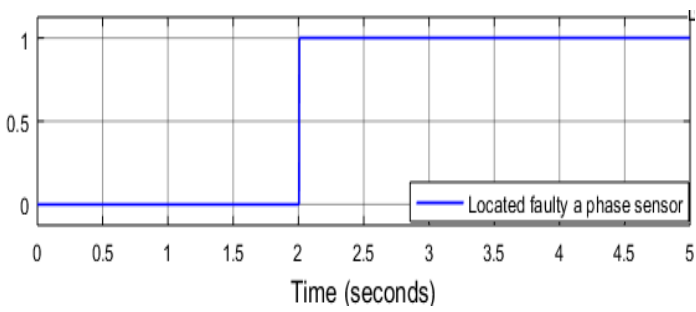

(c)

Fig. 11: Low-speed range: IMD applying axes transformation-FTC method against a current sensor fault: (a) two-phase measured current, (b) fault indication function, (c) rotor speed.

FTC space vector method is shorter than the axes transformation method, as in Fig. 14. At the time of the current fault, the estimated stator current is corrected by the healthy-current sensor, so the estimated signals have high accuracy and small deviation as shown in Fig. 15. The result as, the fluctuation of rotor speed in the proposed method at fault occurrence time is smaller than the axes transformation method, and the IMD operates smoother in the transient period.

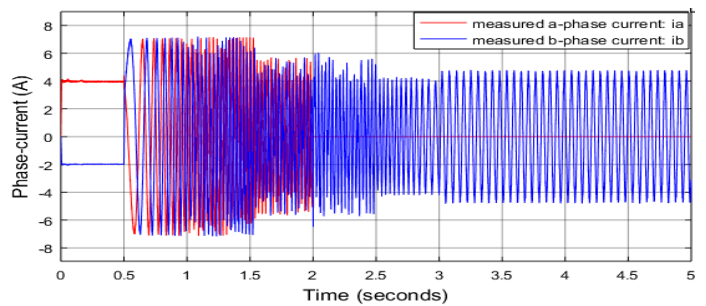

(a)

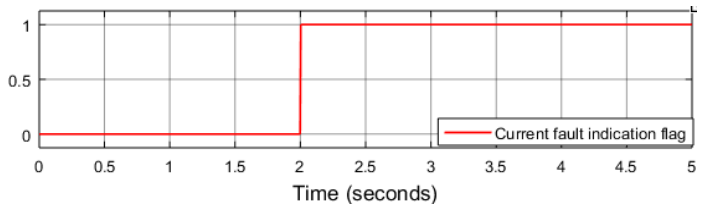

(b)

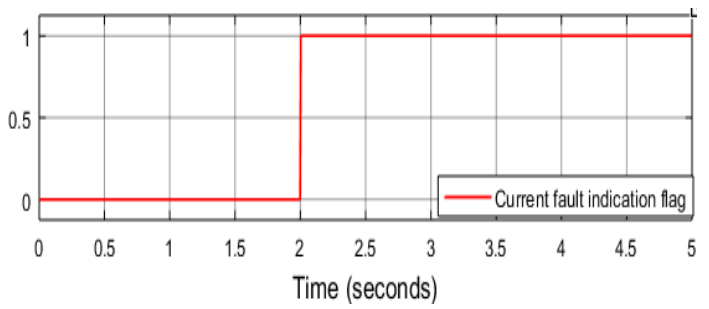

(c)

Fig. 12: Nominal speed range: IMD applying space vector-FTC method against a current sensor fault: (a) two-phase measured current, (b) fault indication function, (c) rotor speed.

\section{Conclusions}

This paper has proposed the upgrading of the FTC method by applying space vectors. This proposed FTC method only uses one observer for fault sensor diagnosis and reconfiguration function. Due to the diagnosis algorithm's simplicity, so the fault detection time of the proposed method is shorter than the axestransformation method, and the IMD system stably operates again quickly. The estimated current has a high precision due to the correction with the signal of the healthy current sensor. Therefore, the proposed method contributes effectively to the development of the FTC technique against the total failure of the current sensor. 


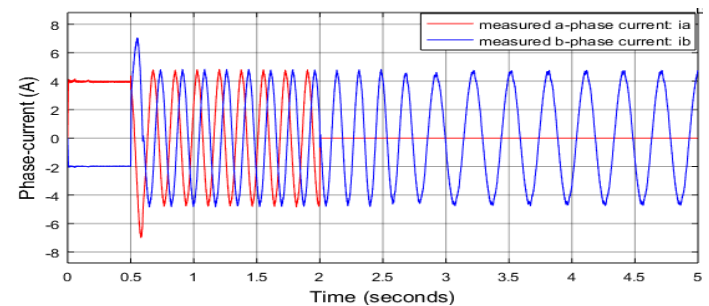

(a)

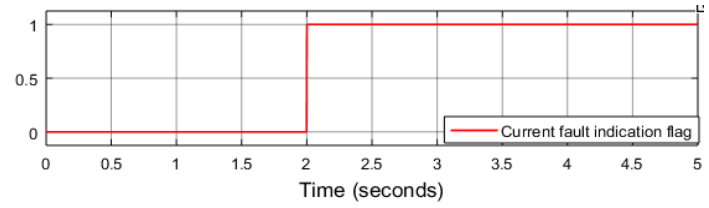

(b)

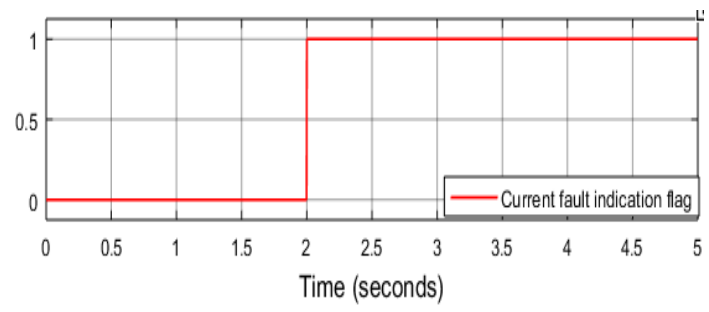

(c)

Fig. 13: Low-speed range: IMD applying space vectorFTC method against a current sensor fault: (a) two-phase measured current, (b) fault indication function, (c) rotor speed.

\section{Acknowledgement}

This research is funded by Graduate Scholarship for Master and Doctoral Programs of Ton Duc Thang University, website: http://grad.tdtu.edu.vn. This work was supported by Project reg. no. SP2020/128 Research and development of sophisticated control methods for the area of electric controlled drives of VSB-Technical University of Ostrava, 2020.

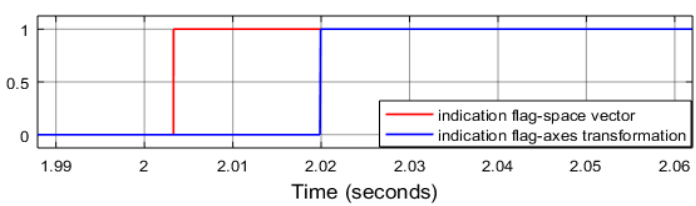

(a)

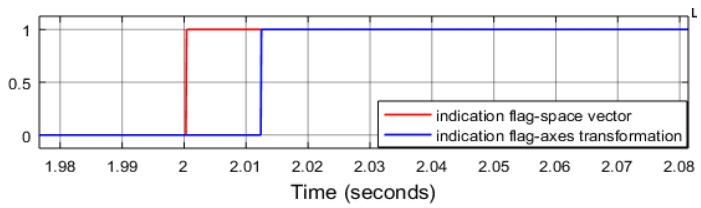

(b)

Fig. 14: Comparison of detection time between the axes transformation-FTC and space vectorFTC method in (a) nominal speed range and (b) low-speed range.

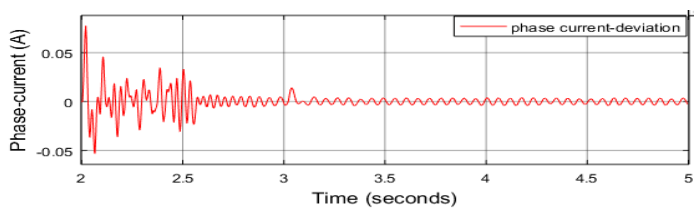

(a)

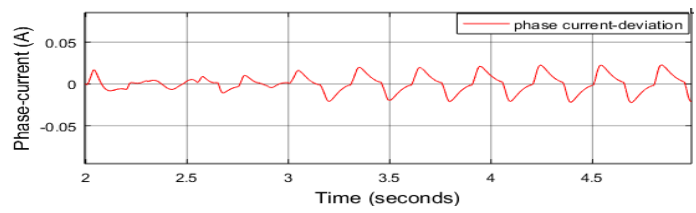

(b)

Fig. 15: The deviation between the estimated current and measured current of the healthy sensor in (a) nominal speed range and (b) low-speed range.

\section{References}

[1] Regaya, C. B., Farhani, F., Zaafouri, A., \& Chaari, A. (2018). A novel adaptive control method for induction motor based on Backstepping approach using dSpace DS 1104 control board. Mechanical Systems and Signal Processing, 100, 466-481.

[2] Liu, Y., Stettenbenz, M., \& Bazzi, A. M. (2018). Smooth Fault-Tolerant Control of 
Induction Motor Drives with Sensor Failures. IEEE Transactions on Power Electronics, 34(4), 3544-3552.

[3] Najafabadi, T. A., Salmasi, F. R., \& Jabehdar-Maralani, P. (2010). Detection and isolation of speed-, DC-link voltage-, and current-sensor faults based on an adaptive observer in induction-motor drives. IEEE Transactions on Industrial Electronics, 58(5), 1662-1672.

[4] Klimkowski, K., \& Dybkowski, M. (2016). A fault tolerant control structure for an induction motor drive system. automatika, 57(3), 638-647.

[5] Chakraborty, C., \& Verma, V. (2014). Speed and current sensor fault detection and isolation technique for induction motor drive using axes transformation. IEEE Transactions on Industrial Electronics, 62(3), 1943-1954.

[6] Baghli, L., Poure, P., \& Rezzoug, A. (2005, September). Sensor fault detection for fault tolerant vector controlled induction machine. In 2005 European Conference on Power Electronics and Applications (pp. 10pp). IEEE.

[7] Yu, Y., Zhao, Y., Wang, B., Huang, X., \& Xu, D. (2017). Current sensor fault diagnosis and tolerant control for VSI-based induction motor drives. IEEE Transactions on Power Electronics, 33(5), 4238-4248.

[8] Rkhissi-Kammoun, Y., Ghommam, J., Boukhnifer, M., \& Mnif, F. (2019). Two current sensor fault detection and isolation schemes for induction motor drives using algebraic estimation approach. Mathematics and Computers in Simulation, 157, 39-62.

[9] Kohlrusz, G., \& Fodor, D. (2011). Comparison of scalar and vector control strategies of induction motors. Hungarian Journal of Industry and Chemistry, 39(2), 265-270.

[10] Melentjev, S., Belahcen, A., Kallaste, A., \& Vaimann, T. (2014, October). Overview of control methods of reluctance machines with sensors and sensorless. In 2014 55th International Scientific Conference on Power and Electrical Engineering of Riga Technical University (RTUCON) (pp. 26-29). IEEE.

[11] Brandstetter, P., \& Kuchar, M. (2015, May). Rotor flux estimation using voltage model of induction motor. In 2015 16th International Scientific Conference on Electric Power Engineering (EPE) (pp. 246250). IEEE.

[12] Brandštetter, P. (2012). Sensorless control of induction motor using modified MRAS. In International Review of Electrical Engineering (I.R.E.E), 7(3), 4404-4411.

[13] Tran, C. D., Brandstetter, P., Ho, S. D., Tran, T. C., Nguyen, M. C. H., Phan, H. X., \& Dinh, B. H. (2018, September). Improving Fault Tolerant Control to the one current sensor failures for induction motor drives. In International Conference on Advanced Engineering Theory and Applications (pp. 789-798). Springer, Cham.

[14] Tran, C. D., Brandstetter, P., Dinh, B. H., Ho, S. D., \& Nguyen, M. H. C. (2018). Current-Sensorless Method for Speed Control of Induction Motor Based on Hysteresis Pulse Width Modulation Technique. Journal of Advanced Engineering and Computation, 2(4), 271-280.

\section{About Authors}

Cuong Dinh TRAN was born in $\mathrm{Ho}$ Chi Minh, Vietnam. He graduated from the Vietnam National University - Ho Chi Minh City - University of Technology. He received his B.E. and M.E. degrees in electrical - electronics power engineering in 2005 and 2008, respectively. Now, he is teaching at the department of electrical and electronics engineering, Ton Duc Thang University, Ho Chi Minh city, Vietnam. His research interests include modern control methods of electrical drives, automatic control system, intelligent control system, operation and control power system. 
Pavel BRANDSTETTER was born in Ostrava, Czech Republic, 1955, 1 June. He received the M.Sc. and Ph.D. degrees in Electrical Engineering from Brno University of Technology, Czech Republic, in 1979 and 1987, respectively. He is currently full professor in Electrical Machines, Apparatus and Drives and dean of Faculty of Electrical Engineering and Computer Science at VSBTechnical University of Ostrava. His current research interests are applied electronics and modern control methods of electrical drives. Since 2000 he has performed research in field of modern control methods for AC motors, for example sensorless control, applications of artificial intelligence in control of $\mathrm{AC}$ drives.

Minh Chau Huu NGUYEN was born in Binh Duong, Vietnam. He graduated from the Military Technical Academy, Vietnam. He received his M.E. degrees in Automation engineering in 2012. Now, he is researching at the Faculty of Electrical Engineering \& Computer Science, VSB - Technical University of Ostrava, Czech Republic. His research interests include an automatic control system, intelligent control system and electrical machine, apparatus and drives.

Sang Dang HO received his B.Eng. and M.Eng degrees in Electrical Engineering from Ho Chi Minh University of Technology, Ho Chi Minh city, Vietnam in 2001 and 2008, respectively. Now, he is teaching at department of electrical and electronics engineering, Ton Duc Thang university, Ho Chi Minh city, Vietnam. His research interests include optimization of power system and electric machines control.

Phuong Nhat PHAM was born in Tien Giang, Vietnam. He graduated from the University of Technical Education Ho Chi Minh City, Viet Nam. He received MSc. degrees in Electrical Equipment, Network and Power Station in 2006. Now, he is teaching at the department of electrical and electronics engineering, Ton Duc Thang University, Ho Chi Minh City, Vietnam. His research interests include Evolutionary algorithm, intelligent control system, operation and control power system.

Bach Hoang DINH received the Ph.D degree in Electrical Engineering from HeriotWatt University, Edinburgh, United Kingdom in 2009. He received the B.E. and the M.E. degrees in Electrical Engineering from Vietnam National University - Hochiminh City in 1995 and 1998, respectively. Bach Dinh is currently the head of Electrical Engineering Department, Faculty of Electrical-Electronic Engineering at Ton Duc Thang University. His research interests are intelligent and optimal control, computer vision, robotics, power electronics, SCADA and industrial communication networks. He is a member of the IEEE Industrial Electronics Society. 